\title{
La Enfermería Transcultural: una intervención con el alumnado
}

\author{
Rocío DE DIEGO CORDERO \\ Universidad de Sevilla \\ Departamento de Enfermeria \\ rdediego2@us.es \\ D.O.I.: http://dx.doi.org/10.12795/JDU.2018.i01.09 \\ Pp.: 161-176
}

\section{Resumen}

Se llevó a cabo un ciclo de mejora en la asignatura Historia, Teoría y Métodos de la Enfermería I de 1o Grado Enfermería, llevando a cabo sesiones en el trabajo en grupos pequeños (seminarios) donde se incrementó la participación del alumnado mediante la realización de esquemas de contenidos y la resolución de casos clínicos, incorporando además la evaluación de ideas previas que ayudaron a orientar las sesiones. Los resultados muestran cómo a pesar de manejar los conceptos teóricos, 3 de los 12 alumnos totales no fueron capaces de aplicarlos a los casos clínicos, lo que supone una oportunidad de mejora.

Palabras clave: Historia, teoría y métodos de la Enfermería I, Grado en Enfermería, Docencia Universitaria, Experimentación Docente Universitaria, Enfermería Transcultural. 


\section{Breve descripción del contexto}

La intervención se hizo con un subgrupo de la asignatura Historia, Teoría y Métodos de la Enfermería I de 10 Grado Enfermería, durante el curso académico 2017/2018. Los contenidos teóricos de esta asignatura pretenden que el alumnado:

- Identifique y relacione el concepto de salud y los cuidados, desde una perspectiva histórica comprendiendo la evolución del cuidado de enfermería.

- Explique desde una perspectiva ontológica y epistemológica, la evolución de los conceptos centrales que configuran la disciplina de enfermería.

- Identifique y diferencie desde una perspectiva ontológica y epistemológica los modelos teóricos más relevantes de la disciplina de enfermería.

A ello hay que unir las competencias trasnversales que se pretenden adquieran con el curso de la misma, tales como el compromiso ético, la capacidad de crítica y autocrítica la habilidad para trabajar de forma autónoma, la capacidad para aplicar la teoría a la práctica, la capacidad de aprender, la solidez en los conocimientos básicos de la profesión, las habilidades para trabajar en grupo y la capacidad de análisis y síntesis.

Tal y como figura en el proyecto docente, los contenidos están distribuidos en 3 bloques:

- Bloque I: historia de la enfermería.

- Bloque II : epistemológia de la disciplina enfermera: teorías y modelos.

- Bloque III: el modelo de necesidades de Virginia Henderson.

En lo referente a las actividades formativas, se contemplan 28 horas de clases teóricas presenciales y 37,5 horas no presenciales. La metodología de enseñanza-aprendizaje 
es la magistral participativa. Las competencias que desarrolla el alumnado son:

- Identificar, integrar y relacionar el concepto de salud y los cuidados, desde una perspectiva histórica, para comprender la evolución del cuidado de enfermería.

- Comprender desde una perspectiva ontológica y epistemológica, la evolución de los conceptos centrales que configuran la disciplina de enfermería, así como los modelos teóricos más relevantes, aplicando la metodología científica en el proceso de cuidar y desarrollando los planes de cuidados correspondientes.

$\begin{array}{cccccc}\text { Ello va unido a exposiciones y } & \text { se- } \\ \text { minarios, } & \text { que suponen un total de } & 22\end{array}$ horas presenciales y 37,5 horas no presenciales. La metodología de enseñanza-aprendizaje se enfoca desde la perspectiva de la enseñanza en grupos pequeños y pretenden completar la formación del estudiante, haciendo énfasis en un aprendizaje concreto, estimulando la enseñanza activa.Las competencias que desarrolla el alumnado son:

- Identificar, integrar y relacionar el concepto de salud y los cuidados, desde una perspectiva histórica, para comprender la evolución del cuidado de enfermería.

- Comprender desde una perspectiva ontológica y epistemológica, la evolución de los conceptos centrales que configuran la disciplina de enfermería, así como los modelos teóricos más relevantes, aplicando la metodología científica en el proceso de cuidar y desarrollando los planes de cuidados correspondientes.

El sistema de evaluación de la asignatura es:

Los contenidos teóricos tiene el $80 \%$ de la nota total y corresponde a:

- Examen escrito: 6 preguntas cortas con un valor de 0,5 puntos y 4 preguntas de desarrollo con un valor de 1 punto.

*La nota teórica máxima es de 7 puntos. 
- Actitud del alumno: con un valor máximo de 1 punto, por la: asistencia y participación del alumno en las clases; esta será sumatoria a la nota teórica una vez aprobada esta.

\section{Para los contenidos de los seminarios:}

Para los contenidos de los seminarios corresponde el $20 \%$ de la nota total 1 . Cada seminario tiene un valor numérico entre 0,15 y 0,2

La asistencia a estos es obligatoria y se evalúa la participación e implicación del alumno en ellos. La nota máxima es de 2, esta será sumatoria a la nota teórica una vez aprobada esta.

En este ciclo de mejora se trabajó con un grupo de 12 alumnas y alumnos, de edades entre los 18 y los 20 años, procediendo en su totalidad del bachillerato. Trabajan divididos en 3 grupos de 4 alumnos/as.

\section{Diseño previo del Ciclo de Mejora Docente}

El diseño que estaba establecido antes de realizar los ciclos de mejora era:

- Parte 1: Presentación por parte del docente del modelo teórico y resumen de los aspectos más relevantes de su modelo (15 min. aprox.).

- Parte 2: Lectura del caso y extracción de los datos para trabajarlo desde la perspectiva de la teórica correspondiente (45-60 min. aprox.)

- Parte 3: Puesta en común de los tres subgrupos (4560 min. aprox.)

A continuación se pasa a elaborar un mapa de contenidos por considerarlo una herramienta de apoyo para organizar los conceptos mas relevantes. (Porlán, 2017). 


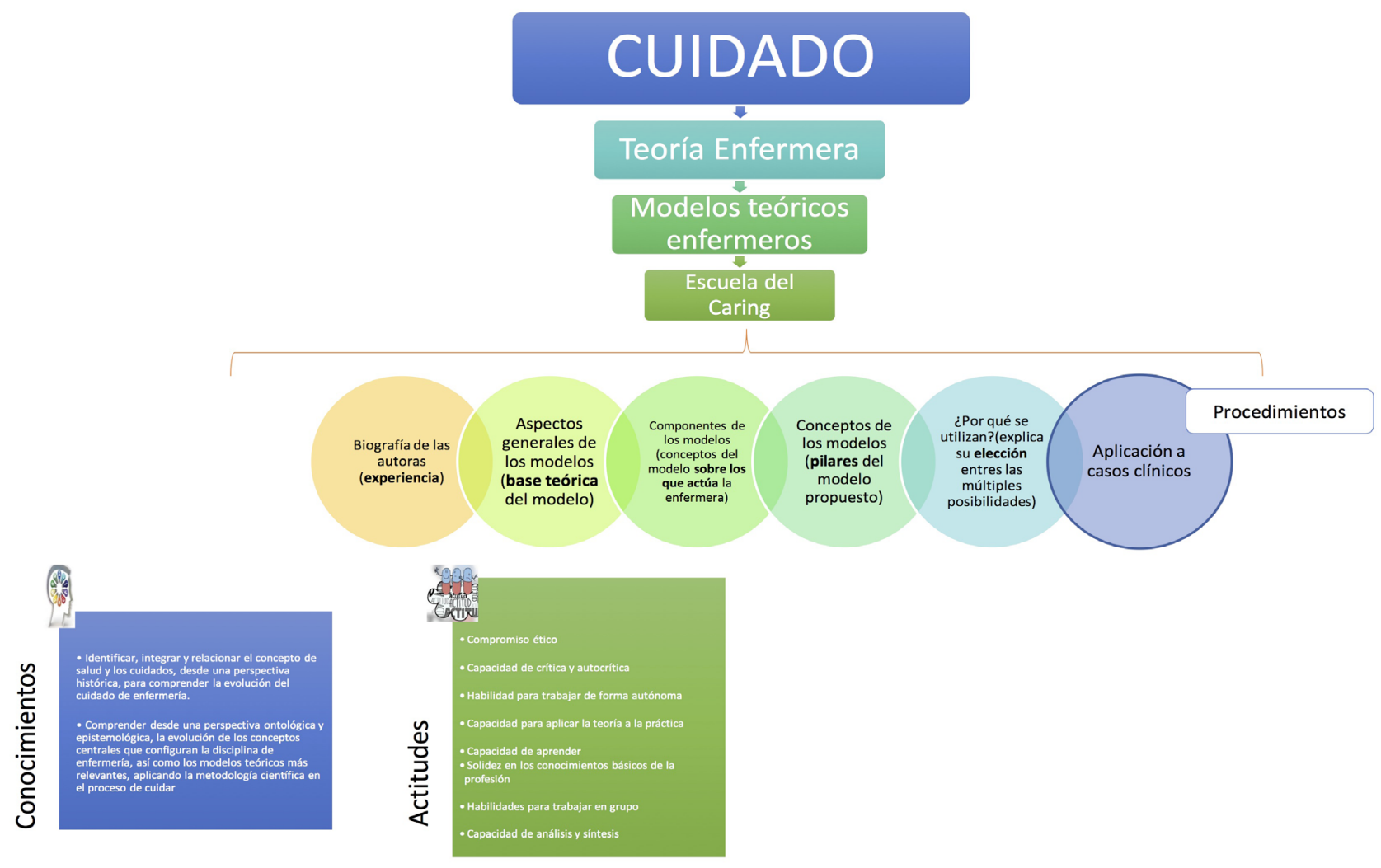

Figura 1: Mapa de contenidos. Elaboración propia

Jornadas de Formación e Innovación Docente del Profesorado | № 1 (2018)

(c) (1) Esta obra se distribuye con la licencia Creative Commons

(c) $(7) \Theta$ Reconocimiento-NoComercial-SinObraDerivada

Internacional (CC BY-NC-ND 4.0.) 
Tras realizar el mapa de contenidos se evidencia que con este modelo hay aspectos tanto de conceptuales, como actitudinales y procedimentales que pueden no estar desarrollándose con este modelo.

Seguidamente se pasa a elaborar el modelo metodológico posible y secuencia de actividades programada, partiendo de los problemas seleccionados. Se presenta a continuación:

Parte 1: Presentación del modelo teórico y resumen de los aspectos más relevantes de su modelo (15 min. aprox.). La propuesta del primer ciclo de mejora fue incorporada en la primera parte, en la que se le ha pedido a cada subgrupo que elabore un esquema previo con los principales conceptos del modelo teórico estudiado, sustituyendo al resumen de los aspectos relevantes que hace el/la docente. Después, al azar, un componente del subgrupo elegido, sale a la pizarra y reproduce el esquema elaborado, explicándolo.

Parte 2: Lectura del caso y extracción de los datos para trabajarlo desde la perspectiva de la teórica correspondiente (45-60 min. aprox.)

Parte 3: Puesta en común de los tres subgrupos (45-60 min. aprox.)

Finkel (2008) plantea empezar con el planteamiento de un problema que despierte el interés del alumnado y para cuya resolución de debe seguir un razonamiento ordenado. Otro de los aspectos de los que habla el autor es el de confiar en que los estudiantes experimenten (y asumir los errores) y diseñar un entorno que los conduzca en la resolución del problema, ya que un alumno que se encuentra superado por el problema puede decidir abandonar la tarea, siendo este el efecto contrario al deseado. Otro aspecto es el tema del trabajo en grupo; la capacidad de trabajar en equipo, de negociar y decidir los pasos es algo para mí fundamental; por ello, trabajo con el alumnado por subgrupos de 3-4 alumnos. 
Así, se decide tratar el tema de la Enfermería Transcultural ya que actualmente los cuidados de enfermería van dirigidos al bienestar de la persona tal y como la persona lo describe. Es decir, los cuidados son individualizados por lo que es imprescindible respetar valores culturales y creencias propias para el éxito del cuidado. Este hecho es lo que diferencia a la enfermería de otras disciplinas y lo que confiere el valor de las tareas que se llevan a cabo (Sanabria, Otero y Urbina, 2002). Y se consideró que estos conceptos podían ser de gran interés para el alumnado.

Ante un entorno que ha sufrido un gran cambio demográfico, en el que coexisten numerosas y variadas normas culturales, el modelo de enfermería transcultural que nos ofrece Madeleine Leininger es un recurso muy útil para que el personal de enfermería explore, conozca, entienda y tenga en cuenta el punto de vista cultural de su paciente. Valorar las creencias y valores culturales de nuestros pacientes perpetua una asistencia satisfactoria, efectiva y coherente. El cuidado transcultural nació en 1960 de la mano esta teórica cuando a raíz de su experiencia clínica definió el cuidado basado en la cultura, creencias y valores. Era necesario instaurar la enfermería transcultural porque además de la realidad del fenómeno de crecimiento cultural de las poblaciones cada vez había más necesidades sentidas de cuidados coherentes con la cultura y los choques culturales eran una barrera que influía negativamente en la salud. Tras años de experiencia Madeleine Leininger se dio cuenta de que las enfermeras no habían recibido educación en temas culturales y que por tanto no eran profesionales eficaces en la práctica. Con base en la enfermería transcultural, Madeleine Leininger enuncia su "Teoría de la diversidad y universalidad de los cuidados". (Leno, 2006) Esta teoría resalta la importancia de explorar los propios conocimientos y experiencias (émic) para después diferenciarlos con factores del medio o punto de vista cultural del paciente (étic). El objetivo del cuidado transcultural es brindar cuidados de acuerdo a las creencias, 
valores y prácticas culturales. Estos cuidados coherentes con la cultura proporcionan bienestar y ayudan a la persona a afrontar su situación de enfermedad. Por tanto el cuidado para Leininger es universal pero las acciones serán diferentes y variables (diversidad) de acuerdo a la cultura que se atienda. En el cuidado cultural Leininger habla de aspectos como la conservación, la negociación/adaptación y el rediseño. La conservación comprende acciones que ayudan a la persona a preservar sus valores culturales para alcanzar el bienestar o morir dignamente; la negociación reúne actividades profesionales que buscan creativamente adaptarse a la persona que recibe los cuidados para obtener buenos resultados en salud; y el rediseño comprende acciones llevadas a cabo para modificar el estilo de vida del usuario respetando siempre sus valores culturales buscando hábitos más saludable.

A partir de los estudios antropológicos, investigaciones y experiencia en enfermería cultural, Leininger elaboró importantes predicciones (Leno, 2006):

- Si los profesionales trabajan con en culturas que les parece muy extrañas y manejan valores diferentes a los esperados culturalmente se producirán conflictos, choques y tensiones con los pacientes.

- Cuanta más dependencia del material tecnológico tenga la labor de enfermería, más importante será el distanciamiento interpersonal y menor satisfacción tendrá el paciente de su atención sanitaria.

- Los cuidados de enfermería que contemplan y se adaptan a prácticas culturales específicas producen satisfacción y bienestar en los usuarios

Tal fue la importancia que la teoría de los cuidados transculturales supuso para la corriente de pensamiento de la disciplina enfermera que esta perspectiva cultural la tomaron como referencia muchos autores, entre ellos Margaret Jean Watson, máxima representante de la escuela del Caring. Watson en este caso nos habla del Cuidado 
Transpersonal, una forma de recuperar el equilibrio entre mente-cuerpo y alma. Se consigue con mutuo compromiso enfermera-paciente, siempre con las emociones presentes (Izquierdo, 2015) y respetando al ser humano en su individualidad (Rodrigues Dos Santos et al, 2014). Visto así el cuidado solo ocurre en la interacción entre dos o más personas y apunta a un nivel superior a las necesidades fisiológicas o el cuerpo físico Esta interacción mejora el proceso de cuidar, transforma y supone abrirse a dimensiones espirituales y existenciales, al alma ligada a la dimensión corporal (Favero et al, 2009). Por ello, son necesarias otras cualidades como la empatía, bondad, comprensión ya que se produzca o no la curación, lo que siempre se da es la atención desde un punto de vista humanístico (Olivé e Isla, 2015). Dicho de otra forma, la enfermera debe de preocuparse de entender el comportamiento del paciente viéndolo desde el mismo punto de vista que este (Zabala et al, 2014). El cuidado tiene carácter contextual, por lo que es imprescindible que la enfermera conozca el ambiente que rodea al individuo (Urra, Jana y García, 2015). Esta forma de cuidar es lo que aporta esencia a la labor de enfermería y reconocimiento a la disciplina.

Para complementar su teoría, Watson define 10 factores asistenciales básicos (Watson lo denomina Proceso Caritas de (uidados) en la labor de la enfermera. Estos diez factores ayudan a diferenciar un cuidado profesional de otro que no lo es:

1. "Práctica de amorosa bondad y ecuanimidad en el contexto de un cuidado consciente": la enfermera mediante la introspección crecerá moralmente y aplicará el desarrollo moral junto con el conocimiento científico.

2. "Ser auténticamente presente y permitir y mantener el sistema de creencias profundas y subjetivas del individuo, compatible con su libertad. Este individuo libre, con creencias propias, es un ser para el cuidado": Permitir que los individuos 
sean fieles a sus creencias y practiquen la espiritualidad.

3. "El cultivo libre de las propias prácticas espirituales y transpersonales, que vaya más allá del ego y la apertura a los demás con sensibilidad y compasión": permitir los propios sentimientos y de los demás, sean emociones negativas o positivas.

4. "Desarrollo y mantenimiento de una auténtica relación de cuidado, a través de una relación de confianza": posibilitar la apertura de los sentimientos y ser honestos con la realidad ya que el ser humano ante todo es único y en menor o mayor grado, autónomo.

5. "Estar presente y constituirse en apoyador de la expresión de los sentimientos positivos y negativos en una conexión con la espiritualidad más profunda del ser que se cuida": permitir la expresión de los sentimientos, como una herramienta que permite contemplar emociones mucho más profundas.

6. "El uso creativo de uno mismo, como partícipe en el arte de cuidar y de todas las maneras de conocer como parte del proceso asistencia": no mecanizar la asistencia y desarrollar habilidades nuevas que no delimiten el campo del quehacer enfermero.

7. "Participar de una verdadera enseñanza-aprendizaje que atienda a la unidad del ser y de su sentido y que trate de mantenerse en el marco referencial del otro": Llevar a cabo educación en salud que permita al usuario empoderarse de su propio proceso de salud/enfermedad.

8. "Creación de un medio ambiente de cuidado en todos los niveles (físico, no físico, ambiental de energía y de conciencia) ya sea el todo, belleza, confort, dignidad y paz": en este medio ambiente surgen variables que Watson llama necesidades, 
directamente relacionadas con la salud del individuo.

9. "La asistencia en necesidades básicas, con un cuidado consciente e intencional que toca y envuelve el espíritu de los individuos, honrando la unidad del Ser, y permitiendo a la espiritualidad emerger": Abordar las necesidades desde un punto de vista integral y holístico.

10. "El reconocimiento de fuerzas fenomenológicas y existenciales": muy importante para la enfermería ya que ayuda al paciente a resolver incongruencias al mismo tiempo que satisface sus necesidades

Para comenzar a tratar estos conceptos con el alumnado se introduce un Test de conocimientos previos con:

- Preguntas: Cuál es tu cultura?/¿Qué otras culturas conoces? Di al menos 2./¿Piensas que hay aspectos de la cultura que pueden influir en la salud? Si la respuesta es si, señala al menos $2 . /$

- Imágenes: observa esta imagen y rellena los bocadillos con lo que pienses pueden estar pensando tanto la enfermera como el paciente en esta situación.

Puede verse el test en la siguiente imagen:

Jornadas de Formación e Innovación Docente del Profesorado | № 1 (2018) Esta obra se distribuye con la licencia Creative Commons 
Test exploración de ideas previas

1. ¿Qué es la cultura?

2. ¿Cuál es tu cultura?

3. ¿Qué otras culturas conoces? di al menos 2.

4. ¿Piensas que hay aspectos de la cultura que pueden influir en la salud? Si la respuesta es si, señala al menos 2.

5. Observa esta imagen y rellena los bocadillos con lo que pienses pueden estar pensando tanto la enfermera como el paciente en esta situación.

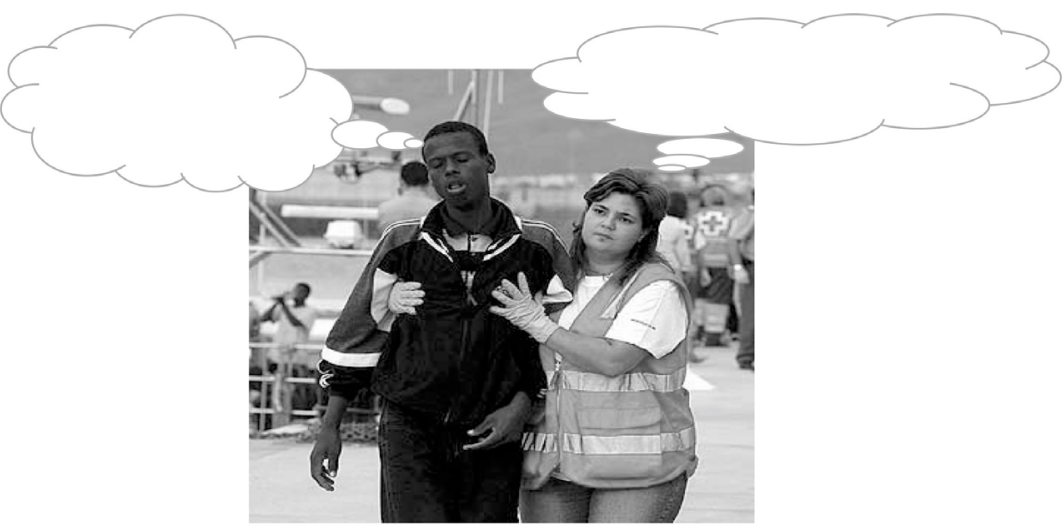

Figura 2: Cuestionario de conocimientos precios

\section{Aplicación del CMD}

Al introducir la propuesta de la elaboración de los esquemas ya en la 5 a sesión, al principio el alumnado se muestra reticente pero una vez le explico mediante ejemplos la tarea se van mostrando más colaborativos. Al final han llevado a cabo los esquemas correctamente y lo han agradecido de cara a que vayan a ser una herramienta a la hora de estudiar la materia.

Jornadas de Formación e Innovación Docente del Profesorado I № 1 (2018)

(c) Esta obra se distribuye con la licencia Creative Commons 
En lo referente a tocar el tema de la cultura entre al alumnado con el test de ideas previas, esto generó un rico debate en el que se encontraron libres de expresar su punto de vista; también es destacable la imagen del inmigrante compartida por el grupo (pobre, sin recursos). Fue una sesión muy provechosa, donde pude conocer qué pensaba el grupo de un aspecto tan importante y donde el alumnado fue consciente de la complejidad de la atención enfermera.

Tras llevar a cabo el análisis de los test, la totalidad reconoce su propia cultura, reconoce otras culturas, cree que hay elementos culturales que pueden afectar a la salud y reconoce la importancia de trabajar desde la perspectiva culturalmente competente.

Tras esto, se le entregan casos clínicos en los que deben aplicar lo comentado en el test; y es aquí donde se aprecia una dificultad de la mayoría, siendo sólo 3 alumnos $(25,1 \%)$ los capaces de resolver adecuadamente esta parte.

Se establecen por tanto 3 modelos de respuesta:

A. Reconoce su propia cultura; reconoce otras culturas; cree que sin duda hay elementos culturales que pueden afectar a la salud. Reconoce la importancia de trabajar desde la perspectiva culturalmente competente. Lo aplica al caso clínico.

B. Reconoce su propia cultura; reconoce otras culturas; cree que hay elementos culturales que pueden afectar a la salud. Reconoce la importancia de trabajar desde la perspectiva culturalmente competente. NO lo aplica al caso clínico.

C. Reconoce su propia cultura; reconoce otras culturas; cree que puede haber elementos culturales que pueden afectar a la salud. No reconoce la importancia de trabajar desde la perspectiva 
culturalmente competente. NO lo aplica al caso clínico.

Modelo C: 16,6\% ALUMNADO

(2 alumnos)

Modelo B: 58,3\% ALUMNADO

(7 alumnos)

Modelo A:25,1 \% ALUMNADO

(3 alumnos)

Figura 3: escalera de aprendizaje. Elaboración propia.

\section{Evaluación del CMD}

En cuanto al aprendizaje de los/as alumnos/as, a pesar de que la interacción con los alumnos es algo habitual en mi práctica, con este curso he aprendido a participar mas pero con "la boca cerrada", dejándole mucho más peso al alumnado.

En cuanto a los contenidos de enseñanza, siendo consciente del escaso margen de maniobra con el que por diversas razones cuento, si que la elaboración del mapa de contenidos me ha servido de guía y ayuda para centrarme en lo importante.

En cuanto a la metodología y actividades, incorporar el test de ideas previas con imágenes y viñetas ha sido algo nuevo para mi que me ha servido muchísimo, que cambia por completo la manera en la que nos enfrentamos a los contenidos y que estoy segura seguiré utilizando.

En cuanto a la evaluación: en lo que se refiere a exámenes, etc. no me ha sido posible insertar ningún cambio, pero si me ha servido para evaluar la dinámica de las clases y plantear cambios y mejoras de cara al próximo curso.

La verdad es que aunque se acerca, este no es fielmente mi modelo didáctico personal pero es verdad que a grandes rasgos contiene muchos de los ingredientes del modelo que me gustaría utilizar. Me ha servido para reflexionar acerca de muchas cuestiones que damos por 
sentadas y que se refieren al interés y preferencia del alumnado y que sin duda condicionan nuestra manera de enseñar.

\section{Principios didácticos argumentados que han guiado la experiencia y que deben permanecer en el futuro.}

- Se diseñará previamente a cada modelo teórico enfermero un test de ideas previas para poder adaptar los contenidos al nivel de conocimiento del alumnado, conocer los puntos de interés y atender a la diversidad.

- Se elaborarán mapas conceptuales previos que ayuden a ordenar y jerarquizar los contenidos a impartir.

- Se utilizarán temas de actualidad o alguna pregunta que despierte el interés del alumnado a la vez que fomente la actitud crítica y analítica.

- Se tomarán notas a modo de "diario del profesor" que ayude a la reflexión diaria del desarrollo de las sesiones.

- Se utilizará el modelo de "dar clase con la boca cerrada" en el que el alumnado será el protagonista, siendo sólo el docente un facilitador y un buen gestor del tiempo.

- Llevar a cabo una evaluación continua de las tareas desarrolladas en las sesiones, las lecturas realizadas y la resolución de los casos, a través de entregas semanales que permitan el feedback con el alumnado y la corrección de errores, facilitando además el aprendizaje.

- Incluir la autoevaluación del alumnado en la práctica docente, además de la evaluación de la asignatura (de la parte correspondiente al trabajo en Grupos Pequeños) así como del/ de la docente. 


\section{Referencias bibliográficas}

Bain, K. (2004). Lo que hacen los mejores profesores universitarios. Valencia: Publicaciones Universidad de Valencia.

Finkel, D. (2008). Dar clases con la boca cerrada. Valencia: Publicaciones Universidad de Valencia.

Mora, F. (2017). Neuroeducación: Solo se puede aprender aquello que se ama. Madrid: Alianza Editorial.

Porlán, R. (2017). Enseñanza Universitaria, Cómo mejorarla. Madrid: Editorial Morata

Sanabria Triana L, Otero Ceballo M, Urbina Laza O. (2002) Los paradigmas como base del pensamiento actual en la profesión de enfermería. Educ Med Super.

Leno González D.(2006) Buscando un modelo de cuidados de enfermería para un entorno multicultural. Gazeta de Antropología, 22(32).

Izquierdo Machín E.(2015) Enfermería: teoría de Jean Watson y la inteligencia emocional, una visión humana. Rev Cubana Enfermería, 31(3).

Rodrigues dos Santos M, Szylit Bousso R, Vendramim P, Freire Baliza M, Deguer Misko M, Silva L.(2014) The practice of nurses caring for families of pediatric impatients in light of Jean Watson. Rev ESC Enferm USP 48: 80-6.

Favero L, Meier MJ, Ribeiro Lacerda M, de Azevedo Mazza V, Canestraro Kalinowski L.(2009) Jean Watson's Theory of Human Caring: a decade of brazilian publications. Acta Paul Enferm 22(2): 213-8.

Olivé Ferrer MC, Isla Pera MP. (2015) El modelo de Watson para un cambio de paradigma en los cuidados enfermeros. Rev ROL Enferm 38(2): 123-8.

Zavala-Pérez IC, Hernán dez-Corrales MD, Olea-Gutiérrez CV, Valle-Solís MO. (2014) Cuidado de enfermería transpersonal con base en la teoría del caring a una mujer con cáncer de mama. Rev Enferm IMSS mex. 22(3): 135-43.

Urra E, Jana A, García M.(2011) Algunos aspectos esenciales del pensamiento de Jean Watson y su teoría de cuidados transpersonales. Cienc enferm 17(3): 11-22.

Jornadas de Formación e Innovación Docente del Profesorado | № 1 (2018) Esta obra se distribuye con la licencia Creative Commons Reconocimiento-NoComercial-SinObraDerivada Internacional (CC BY-NC-ND 4.0.) 